\section{OPEN ACCESS}

Edited by:

Feng Liu,

Tianjin Medical University General Hospital, China

Reviewed by:

Liting Chen,

The First Affiliated Hospital of Nanchang University, China

Xiaonan Guo,

University of Electronic Science and Technology of China, China

*Correspondence:

Yun Xu

xuyun20042001@aliyun.com

${ }^{\dagger}$ These authors have contributed equally to this work

Specialty section: This article was submitted to Brain Imaging Methods, a section of the journal Frontiers in Neuroscience

Received: 09 January 2019 Accepted: 19 June 2019 Published: 03 July 2019

Citation: Ye $Q$, Chen X, Qin R, Huang L, Yang $D$, Liu $R$, Zhang $B$, Bai F and

Xu Y (2019) Enhanced Regional

Homogeneity and Functional Connectivity in Subjects With White Matter Hyperintensities and Cognitive Impairment. Front. Neurosci. 13:695. doi: 10.3389/fnins.2019.00695

\title{
Enhanced Regional Homogeneity and Functional Connectivity in Subjects With White Matter Hyperintensities and Cognitive Impairment
}

Qing Ye ${ }^{1,2,3 t}$, Xin Chen ${ }^{1+}$, Ruomeng Qin ${ }^{1,2,3}$, Lili Huang ${ }^{1}$, Dan Yang ${ }^{1}$, Renyuan Liu ${ }^{1}$, Bing Zhang ${ }^{4}$, Feng Bai ${ }^{1,2,3}$ and Yun $\mathrm{Xu}^{1,2,3 *}$

${ }^{1}$ Department of Neurology, Affiliated Drum Tower Hospital, Jiangsu Key Laboratory for Molecular Medicine, Nanjing University Medical School, Nanjing, China, ${ }^{2}$ Jiangsu Province Stroke Center for Diagnosis and Therapy, Nanjing, China,

${ }^{3}$ Nanjing Neuropsychiatry Clinic Medical Center, Nanjing, China, ${ }^{4}$ Department of Radiology, Affiliated Drum Tower Hospital, Nanjing University Medical School, Nanjing, China

Objective: White matter hyperintensities $(\mathrm{WMH})$ is an important cause of vascular cognitive impairment $(\mathrm{Cl})$. However, a considerable portion of individuals with $\mathrm{WMH}$ do not develop $\mathrm{Cl}$. The present study aimed to investigate distinctive regional brain activity and connectivity patterns in $\mathrm{WMH}$ subjects with and without $\mathrm{Cl}$, who displayed comparable WMH burden.

Methods: Fourteen WMH subjects with $\mathrm{Cl}, 16 \mathrm{WMH}$ subjects without $\mathrm{Cl}$ and 37 healthy subjects underwent multimodal MRI scans and neuropsychological tests. All WMH subjects displayed Fazekas grade 2 of $\mathrm{WMH}$. Regional Homogeneity (ReHo) and functional connectivity (FC) patterns were identified based on resting-state functional MRI data.

Results: No significant differences in $\mathrm{WMH}$ volume, the number of $\mathrm{WMH}$ lesions and brain volume were shown between the $2 \mathrm{WMH}$ groups. In contrast, the $\mathrm{WMH}$ with $\mathrm{Cl}$ group showed higher ReHo in bilateral superior parietal gyrus (SPG)/superior occipital gyrus (SOG) than the $\mathrm{WMH}$ without $\mathrm{Cl}$ group. Compared with the $\mathrm{WMH}$ without $\mathrm{Cl}$ group, the WMH with $\mathrm{Cl}$ group also displayed higher FC of the left SPG/SOG with frontal regions, and higher FC of the right SPG/SOG with parietal regions. Furthermore, higher FC of the left SPG/SOG with frontal regions were significantly associated with less worse executive dysfunction in WMH with $\mathrm{Cl}$ subjects, suggesting a compensatory effect.

Conclusion: Higher local coherence of activities in the SPG/SOG and higher connectivity of the SPG/SOG with parietal and frontal regions are related to $\mathrm{Cl}$ in $\mathrm{WMH}$ subjects. The findings provide novel insights into functional alterations underlying the cognitive variety in WMH subjects.

Keywords: white matter hyperintensities, cognitive impairment, regional homogeneity, functional connectivity, cognitive variety 


\section{INTRODUCTION}

White matter hyperintensities (WMH), defined in the $\mathrm{T}_{2}$ weighted magnetic resonance imaging (MRI) representation, is widely common in elderly population. The prevalence of $\mathrm{WMH}$ increases remarkably with age and is as high as $72-96 \%$ in population over 60 years old (Longstreth et al., 1996; de Leeuw et al., 2001; Zhuang et al., 2018; Lampe et al., 2019). As a MRI marker of cerebral small vessel disease, the pathology of WMH generally reflects loss of axons and myelin, myelin pallor and gliosis (Gouw et al., 2011). These lesions are associated with lacunar infarction occurrence (Xu et al., 2018), and may disrupt white matter tracts or U-fibers that mediate corticalsubcortical or cortical-cortical connections, thus resulting in cognitive impairment (CI).

A large body of evidence shows that WMH causes vascular CI (Prins et al., 2005; Debette and Markus, 2010; Brickman et al., 2015), and WMH is associated with impairments in executive function and processing speed (Prins et al., 2005; Sudo et al., 2013). The baseline WMH burden was related with an increased risk of developing dementia, and the WMH progression was related with declines in global cognitive function and information processing speed (van Dijk et al., 2008). The progression of WMH correlated better with cognitive decline than did the baseline WMH burden (Schmidt R. et al., 2012). However, WMH is widely common in elderly population and not all subjects with WMH will develop CI. A recent study showed that WMH was detected in $77.8 \%$ healthy elderly between 60 and 82 years old (Lampe et al., 2019). Investigating the mechanisms underlying the link between CI and WMH may help to understand the cognitive heterogeneity in subjects with WMH.

Resting-state (fMRI) techniques have been increasingly utilized to investigate functional alterations related to the onset of $\mathrm{CI}$ in WMH. Recently, a study indicated that Regional Homogeneity (ReHo) in the left cerebellum and the middle cingulate cortex were significantly correlated with CI and executive function deficits respectively in subjects with both CI and WMH (Diciotti et al., 2017). Compared with WMH subjects with normal cognition, WMH subjects with CI displayed lower functional connectivity (FC) of posterior cingulate cortex with anterior cingulate cortex, temporal regions and frontal regions, and higher $\mathrm{FC}$ with specific temporal regions and parietal regions (Sun et al., 2011). Several other studies detected altered FC or brain activation patterns across frontal, parietal, temporal and occipital regions in WMH subjects with normal cognition (Lockhart et al., 2015; De Marco et al., 2017; Shi et al., 2017).

Most of the above researches were performed only in WMH subjects with normal cognition or WMH subjects with CI. These findings did not explain why only a portion of the population with WMH would develop CI. The present study recruited WMH subjects with $\mathrm{CI}, \mathrm{WMH}$ subjects without $\mathrm{CI}$ and healthy subjects, and the $2 \mathrm{WMH}$ groups had comparable WMH burden. We hypothesized that different ReHo and FC patterns would be shown between the WMH with and without CI groups and these functional alterations may be related to the mechanism of WMH-mediated CI.

\section{MATERIALS AND METHODS}

\section{Participants}

The present study was carried out in accordance with the latest version of the Declaration of Helsinki, and approved by the Drum Tower Hospital Research Ethics Committee. Thirtyseven healthy subjects and 30 subjects with WMH (Fazekas grade 2) were recruited at the Drum Tower Hospital, Medical School of Nanjing University. All participants provided written informed consents and underwent multimodal MRI scans and a standardized diagnostic evaluation, including demographic data, medical history and an examination of neuropsychological status.

\section{Neuropsychological Examination}

Global cognitive function was measured using a Montreal Cognitive Assessment (MoCA) and a Mini Mental State Examination (MMSE). WMH subjects with MoCA scores lower than education-adjusted norms (the cutoff was $\leq 19$ for $1 \sim 6$ years of education, $\leq 24$ for $7 \sim 12$ years of education and $<26$ for $>12$ years of education) were defined as the WMH with CI group $(n=14)$, and other WMH subjects were defined as the WMH without CI group $(n=16)$. All subjects underwent a neuropsychological battery test including Trail Making Tests (TMT)-A and B and Stroop Color and Word Tests A, B, and C (Stroop-A, B, and C). Three WMH with CI subjects failed to perform some of tests due to subjective unwillingness or hypopsia. The mental statuses were assessed with the Structured Clinical Interview for Diagnostic and Statistical Manual of Mental Disorders, Fourth Edition (DSM-IV) Axis I Disorders (SCIDI), the Hamilton Anxiety Scale (HAMA), and the Hamilton Depression Scale (HAMD).

\section{Inclusion and Exclusion Criteria}

The inclusion criteria for WMH subjects were as follows: (1) age $>50$ years, (2) the presence of Fazekas grade 2 of WMH on MRI images, (3) possible subjective complaints like memory impairment, postural instability, dizziness or depression. WMH were described as hyperintensities on FLAIR images, without cavitation. Fazekas grade 2 of WMH was defined as single lesions between 1 and $2 \mathrm{~cm}$, areas of "grouped" lesions more than $2 \mathrm{~cm}$ in any diameter, and no more than "connecting bridges" between individual lesions (Pantoni et al., 2005).

Exclusion criteria were as follows: (1) a history of ischemic stroke with infarcts of more than $1.5 \mathrm{~cm}$ in diameter or cardiogenic cerebral infarction, (2) intracranial hemorrhage, (3) carotid artery stenosis $(>75 \%)$ or coronary atherosclerosis heart disease, (4) other neurological disorders, such as Alzheimer disease (AD), Parkinson(ism), epilepsy and multiple sclerosis, (5) systemic disease, such as cancer, shock, anemia and thyroid dysfunction, (6) MRI contraindications, (7) prominent impairments of audition or vision.

\section{MRI Procedures}

Magnetic resonance imaging scanning was performed using a 3 Tesla MR scanner (Achieva 3.0 T Ingenia; Philips Medical Systems, Eindhoven, Netherlands) with a 32-channel head coil at 
the Drum Tower Hospital, Medical School of Nanjing University. All subjects were told to relax, close their eyes and stay awake during scanning. Their heads were immobilized using belts and foam pads to minimize head motion, and their ears were occluded with earplugs. High-resolution $\mathrm{T}_{1}$-weighted sagittal images covering the whole brain were obtained by a $3 \mathrm{D}$ magnetization prepared rapid gradient-echo sequence: repetition time $(\mathrm{TR})=9.8 \mathrm{~ms}$; echo time $(\mathrm{TE})=4.6 \mathrm{~ms}$; field of view $(\mathrm{FOV})=256 \times 256 \mathrm{~mm}$; acquisition matrix $=256 \times 256$; flip angle $(\mathrm{FA})=8^{\circ}$; thickness $=1.0 \mathrm{~mm}$, gap $=0 \mathrm{~mm}$; number of slices $=192$. Resting-state functional images, including 230 volumes, were obtained by a gradient-recalled echo-planar imaging (GRE-EPI) sequence: $\mathrm{TR}=2000 \mathrm{~ms} ; \mathrm{TE}=30 \mathrm{~ms}$; FOV $=192 \times 192 \mathrm{~mm}$; acquisition matrix $=64 \times 64$; $\mathrm{FA}=90^{\circ}$; thickness $=4.0 \mathrm{~mm}$; gap $=0 \mathrm{~mm}$; number of slices $=35$. Additionally, $\mathrm{T}_{2}$ FLAIR axial images were obtained with following parameters: $\mathrm{TR}=4500 \mathrm{~ms}$; $\mathrm{TE}=344 \mathrm{~ms}$; acquisition matrix $=272 \times 272 ; \mathrm{FA}=90^{\circ}$; thickness $=1 \mathrm{~mm}$; gap $=0 \mathrm{~mm}$, number of slices $=200$.

\section{WMH Segmentation and Quantification}

As shown in Figure 1, WMH volume was measured on $\mathrm{T}_{1}$ weighted and $\mathrm{T}_{2}$ FLAIR images using the lesion growth algorithm (Schmidt P. et al., 2012) as implemented in the LST toolbox version 2.0.15 ${ }^{1}$ for Statistical Parametric Mapping software $\left(\mathrm{SPM} 12^{2}\right)$. First, the algorithm segments the $\mathrm{T}_{1}$ images into gray matter, white matter and cerebrospinal fluid. The information is then combined with the coregistered $\mathrm{T}_{2}$ FLAIR intensities to calculate lesion belief maps. By thresholding these maps with a pre-chosen initial threshold $(\kappa=0.30)$, an initial binary lesion map is obtained and is subsequently grown along voxels that appear hyperintense on the $\mathrm{T}_{2}$ FLAIR image. The result is a lesion probability map. It should be noted that the $\kappa$-value was determined through the visual inspection of the results by 3 experienced raters.

\section{Volume of Brain and Hippocampus}

As described in our previous study (Ye et al., 2017), brain volume was assessed using the VBM8 toolbox for SPM12. First, the $\mathrm{T}_{1}$ images were segmented into gray matter, white matter and cerebrospinal fluid. Second, the segmented images were normalized to the MNI template using a non-linear and affine spatial normalization and re-sampled to a voxel size of $1.5 \times 1.5 \times 1.5 \mathrm{~mm}$. Third, Jacobian modulation was applied to the segmented images, which could be incorporated to compensate for the effect of spatial normalization. Fourth, the extracted gray matter, white matter and cerebrospinal fluid sets were smoothed with an $8-\mathrm{mm}$ full width at half maximum Gaussian filter to decrease the effects of individual variation in gyral anatomy and to increase the signal-to-noise ratio. Finally, gray matter volume, white matter volume, cerebrospinal fluid volume and whole brain volume were obtained in each subject.

Hippocampal atrophy is a well-established indicator for the early diagnosis of AD (Aisen et al., 2010; Jack et al., 2010), which

${ }^{1}$ www.statistical-modeling.de/lst.html

${ }^{2}$ http://www.fil.ion.ucl.ac.uk/spm
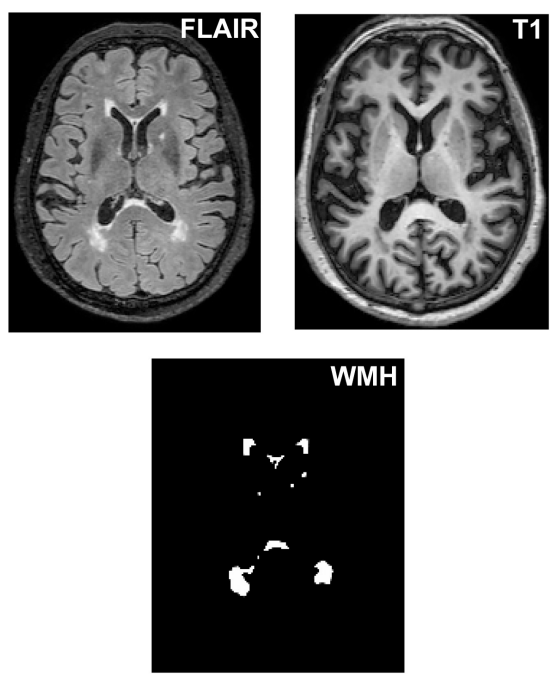

FIGURE 1 | The segmentation of WMH lesions. WMH lesions were segmented and quantified from $T_{2}$ FLAIR images and $T_{1}$ images.

is the most common type of dementia in the elderly population. To rule out the $\mathrm{CI}$ due to $\mathrm{AD}$, the hippocampal volume was assessed. The hippocampus (left and right separately) was isolated using automated anatomical labeling implemented through the Resting State fMRI Data Analysis Toolkit 1.7 software ${ }^{3}$. Then, the hippocampal regions were interpolated to the same sizes, dimension and origins with $\mathrm{T}_{1}$ images. And a mean volume index of all voxels within the hippocampal region (left and right) was extracted for each subject. Finally, the hippocampal volume was obtained by multiplying the mean volume index by the size of each voxel $(1.5 \times 1.5 \times 1.5 \mathrm{~mm})$ and the number of voxels within the hippocampal region.

\section{Resting-State Functional Image Preprocessing}

Functional MRI data were preprocessed using a toolbox for Data Processing and Analysis for Brain Imaging (DPABI) V2.34. Owing to $\mathrm{T}_{1}$ equilibration effects, the first 10 volumes of the scanning session were discarded. The slice timing and realignment procedures were conducted to correct for the time differences in acquisition among slices within one volume, and the motion effects (Friston 24-parameter model) during scanning. A control subject was excluded due to head motion artifacts exceeding $2^{\circ}$ in rotation or $2 \mathrm{~mm}$ in transition. The resulting images were spatially normalized into a standard stereotaxic space with a 12-parameter affine approach and an EPI template image, and then resampled to $3 \times 3 \times 3 \mathrm{~mm}$ voxels, and smoothed with a Gaussian kernel of $6 \times 6 \times 6 \mathrm{~mm}$. Then, white matter signal, cerebrospinal fluid signal and 24 head motion parameters were removed as covariates of no interest. The resulting fMRI data were band-pass filtered $(0.01-0.08 \mathrm{~Hz})$, and the linear trend of time courses was removed. Finally, scrubbing

\footnotetext{
${ }^{3}$ http://restfmri.net/forum/index.php

${ }^{4}$ http://rfmri.org/DPABI
} 
was performed. Volumes with framewise displacement (FD) larger than $0.5 \mathrm{~mm}$ with prior 1 and later 2 volumes were deleted, and subjects with fewer than 4 min of remaining data (about 50\% volumes) were excluded (Power et al., 2012; Satterthwaite et al., 2013; Chen et al., 2019; Guo et al., 2019). After exclusion, 33 control subjects, $14 \mathrm{WMH}$ without CI subjects and $14 \mathrm{WMH}$ with CI subjects remained.

\section{ReHo Analysis}

ReHo analysis was performed without smoothing using a toolbox for DPABI V2.3. According to a previous study (Zang et al., 2004), individual ReHo maps were obtained by calculating the Kendall's coefficient concordance of the time series of a given voxel with those of its nearest neighbors (26 voxels) in a voxelwise manner. To improve the normality and reliability of ReHo value across subjects (Zuo et al., 2013), all individual ReHo maps were standardized into ReHo $z$-value by subtracting the average voxel-wise ReHo obtained for the entire brain, and then dividing the resultant value by the standard deviation. Finally, generated ReHo maps were spatially smoothed with a Gaussian kernel of $6 \times 6 \times 6 \mathrm{~mm}$.

\section{FC Analysis}

Regions showing significant difference of ReHo between the $\mathrm{WMH}$ with $\mathrm{CI}$ group and the WMH without CI group served as seeds for FC analysis. For each subject, a mean time series of each seed region was extracted as a reference time course. Pearson cross-correlation analysis was conducted between the reference time course and time course of each voxel in the brain. Then, a Fisher's $z$-transformation was used to improve the normality of the correlation coefficients $z=0.5 \times \ln \frac{1+\mathrm{r}}{1-\mathrm{r}}$. Finally, the individual FC maps of each region showing significant group difference of ReHo were obtained.

\section{Statistical Analysis} Demographic, Neuropsychological, and Volume Data A one-way analysis of variance (ANOVA) was performed in the analyses of age, education, volume data and mean FD with significance at $P<0.05$ among the control group, the $\mathrm{WMH}$ without $\mathrm{CI}$ group and the WMH with CI group. $\chi^{2}$ test was applied in the analysis of gender among the three groups. Because neuropsychological data was non-normal distribution, the Kruskal-Wallis test was applied in the analyses of neuropsychological data with significance at $P<0.05$ among the three groups. The SPSS 19.0 software (SPSS, Inc., Chicago, IL, United States) was employed in these statistical procedures.

\section{ReHo and FC Data}

The ReHo or FC differences among the three groups were analyzed by applying a voxel-wise one-way analysis of covariance (ANCOVA), controlling for age, gender, years of education and mean FD (using DPABI V2.3). The thresholds were set at a corrected $P<0.01$, determined by Monte Carlo simulation for multiple comparisons (voxel-wise $P<0.01$ ), and FWHM will be estimated to determine the threshold of cluster size. Then, the mean ReHo or FC strength in each significant cluster was extracted in each subject. A post hoc $t$-test was performed to find the detailed between-group ReHo or FC difference in each cluster employing the SPSS 19.0 software. Multiple comparison correction, i.e., the Bonferroni correction principle, was performed for post hoc comparisons. Finally, Pearson correlation analyses were performed between the mean ReHo or FC strength in each cluster and the cognitive test scores in the WMH with CI group using the SPSS 19.0 software with significance at $P<0.05$.

\section{RESULTS}

\section{Demographic and Neuropsychological Data}

As shown in Table 1, no significant differences in age, education and gender were found among the control group, the $\mathrm{WMH}$ without CI group and the WMH with CI group. The WMH with CI group performed significantly worse in MMSE, MoCA, TMT-B, Stroop-B, and Stroop-C tests than both the control group and the WMH without $\mathrm{CI}$ group (all $P<0.05$ ). No significant differences in cognitive test scores were shown between the $\mathrm{WMH}$ without $\mathrm{CI}$ group and the control group.

\section{Volume Data}

As shown in Table 1, both the WMH with CI group and the $\mathrm{WMH}$ without CI group displayed significantly larger total $\mathrm{WMH}$ volume and more number of WMH lesions than the control group (all $P<0.05$ ). Notably, no significant differences in total $\mathrm{WMH}$ volume and the number of $\mathrm{WMH}$ lesions were found between the $\mathrm{WMH}$ with $\mathrm{CI}$ group and the $\mathrm{WMH}$ without CI group. Furthermore, no significant differences in whole brain volume, gray matter volume, white matter volume, bilateral hippocampal volume and mean FD were shown among the three groups.

\section{ReHo Data}

As shown in Figure 2A, the three groups displayed significant differences of ReHo in right superior temporal gyrus/Heschl's gyrus, left superior parietal gyrus (SPG)/superior occipital gyrus (SOG) and right SPG/SOG.

\section{Post hoc Analysis}

In the right superior temporal gyrus/Heschl's gyrus, both the $\mathrm{WMH}$ with CI group and the WMH without CI group displayed lower ReHo than the control group (both $P<0.001$ ), and no significant difference of ReHo was shown between the $2 \mathrm{WMH}$ groups $(P=0.27)$ (Figure 2B). Interestingly, in the left SPG/SOG and right SPG/SOG, the WMH with CI group showed higher ReHo than both the WMH without CI group $(P=0.002$ and $P<0.001$, respectively) and the control group (both $P<0.001$ ) (Figures 2C,D).

\section{FC Data}

Since the $2 \mathrm{WMH}$ groups displayed significant differences of ReHo in the left SPG/SOG and right SPG/SOG, we further investigated the differences of FC pattern of the two regions among the three groups. 
TABLE 1 | Demographic, neuropsychological and volume data.

\begin{tabular}{|c|c|c|c|c|c|}
\hline Items & Control $(n=33)$ & WMH without $\mathrm{Cl}(n=14)$ & WMH with $\mathrm{Cl}(n=14)$ & F or $\chi^{2}$ & $P$-value \\
\hline Age (years) & $62.03 \pm 7.53$ & $63.75 \pm 8.29$ & $66.00 \pm 5.13$ & 1.85 & 0.190 \\
\hline Education (years) & $10.88 \pm 3.49$ & $10.31 \pm 3.89$ & $9.93 \pm 2.89$ & 0.76 & 0.518 \\
\hline Gender (male: female) & $16: 17$ & $8: 6$ & $7: 7$ & 0.30 & 0.861 \\
\hline MMSE & $28.47 \pm 1.49$ & $28.37 \pm 1.32$ & $26.86 \pm 2.66^{a, b}$ & - & 0.034 \\
\hline MoCA & $26.41 \pm 2.30$ & $25.48 \pm 2.38$ & $20.43 \pm 2.71^{a, b}$ & - & $<0.001$ \\
\hline TMT-A & $49.52 \pm 15.87$ & $50.81 \pm 22.99$ & $66.69 \pm 32.71$ & - & 0.092 \\
\hline TMT-B & $82.26 \pm 28.86$ & $110.85 \pm 64.01$ & $155.90 \pm 81.78^{a, b}$ & - & 0.001 \\
\hline Stroop-A & $17.83 \pm 6.11$ & $17.19 \pm 5.17$ & $22.27 \pm 6.56$ & - & 0.079 \\
\hline Stroop-B & $19.97 \pm 7.12$ & $20.75 \pm 6.79$ & $33.55 \pm 19.00^{a, b}$ & - & 0.015 \\
\hline Stroop-C & $29.96 \pm 7.89$ & $30.73 \pm 11.92$ & $52.27 \pm 36.68^{a, b}$ & - & 0.007 \\
\hline Total WMH volume (ml) & $1.28 \pm 1.12$ & $6.06 \pm 2.27^{a}$ & $6.60 \pm 4.25^{a}$ & 37.85 & $<0.001$ \\
\hline Number of WMH lesions & $8.81 \pm 3.53$ & $16.17 \pm 6.02^{a}$ & $15.43 \pm 4.29^{a}$ & 20.12 & $<0.001$ \\
\hline Whole brain volume (ml) & $1239.62 \pm 185.18$ & $1230.48 \pm 169.59$ & $1246.71 \pm 168.05$ & 0.12 & 0.89 \\
\hline Gray matter volume (ml) & $483.74 \pm 75.80$ & $483.63 \pm 72.96$ & $497.57 \pm 62.44$ & 0.21 & 0.721 \\
\hline White matter volume (ml) & $432.87 \pm 78.33$ & $424.62 \pm 69.04$ & $440.21 \pm 63.28$ & 0.29 & 0.788 \\
\hline Left hippocampal volume (ml) & $2.77 \pm 0.39$ & $2.53 \pm 0.38$ & $2.58 \pm 0.28$ & 1.42 & 0.283 \\
\hline Right hippocampal volume (ml) & $2.71 \pm 0.55$ & $2.52 \pm 0.48$ & $2.68 \pm 0.35$ & 0.92 & 0.408 \\
\hline Mean FD (mm) & $0.17 \pm 0.05$ & $0.15 \pm 0.04$ & $0.18 \pm 0.07$ & 1.00 & 0.376 \\
\hline
\end{tabular}

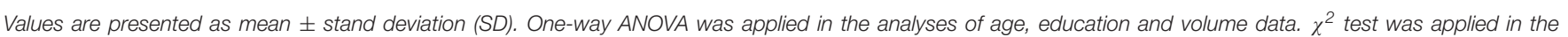

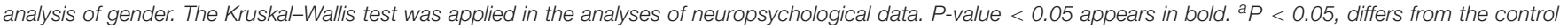

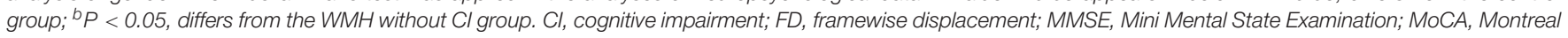
Cognitive Assessment; TMT-A and TMT-B, Trail Making Tests-A and B; WMH, white matter hyperintensities.

As shown in Figure 3A, the three groups displayed significant differences of FC of the left SPG/SOG in the right inferior/middle frontal gyrus, left inferior occipital gyrus and left hippocampus. As shown in Figure $\mathbf{3 B}$, the three groups displayed significant differences of FC of the right SPG/SOG in the bilateral postcentral gyrus/inferior parietal lobule and bilateral hippocampus and thalamus. The detailed coordinate information of above regions was shown in Table 2.

Post hoc analysis: As shown in Table 3, first, for the FC of the left SPG/SOG, the WMH with CI group displayed higher FC than the control group in all regions with significances (all $P<0.001)$. In contrast, the WMH without CI group displayed higher FC than the control group only in the left hippocampus $(P=0.004)$. Notably, the WMH with CI group showed higher FC than the WMH without CI group in the right inferior/middle frontal gyrus $(P<0.001)$. Second, for the FC of the right SPG/SOG, the WMH with CI group displayed higher FC than the control group in all regions with significances $(P<0.001$ for the bilateral postcentral gyrus/inferior parietal lobule, and $P=0.002$ for the bilateral hippocampus and thalamus). Compared with the control group, the WMH without CI group displayed higher FC in the bilateral hippocampus and thalamus $(P=0.003)$. Notably, the WMH with CI group displayed higher FC than the WMH without CI group in the bilateral postcentral gyrus/inferior parietal lobule $(P<0.001)$.

\section{Behavioral Significance of ReHo and FC Alterations}

Correlative analyses between functional brain alterations and cognition were performed in the $\mathrm{WMH}$ with $\mathrm{CI}$ group. As shown in Figures 4A,B, lower ReHo in the right superior temporal gyrus/Heschl's gyrus was significantly associated with longer TMT-B and Stroop-A time $(r=-0.773, P=0.009$ and $r=-0.783, P=0.011$, respectively), i.e., worse executive function and processing speed. As shown in Figures 4C,D, higher FC of the left SPG/SOG with the left hippocampus and the right inferior/middle frontal gyrus was significantly associated with shorter Stroop-A and Stroop-C time, respectively $(r=-0.628$, $P=0.039$ and $r=-0.728, P=0.012$, respectively), suggesting a compensatory effect.

\section{DISCUSSION}

The present study was the first to show the differences in ReHo and FC patterns between WMH with and without CI subjects who had comparable WMH burden. Worse global function, executive function and processing speed were shown in the WMH with CI group. The $2 \mathrm{WMH}$ groups showed no significant differences in brain volume data. However, the WMH with $\mathrm{CI}$ group displayed higher ReHo in the bilateral SPG/SOG than the WMH without CI group. The WMH with CI group also displayed higher FC of the SPG/SOG with parietal and frontal regions. Furthermore, the ReHo and FC alterations were correlated with cognitive function in WMH with CI subjects.

A previous resting-state fMRI study investigated FC patterns in both WMH subjects with normal cognition and WMH subjects with CI, and found altered FC of posterior cingulate cortex with extensive regions in WMH subjects with CI. However, the WMH burden was not evaluated in the two groups (Sun et al., 2011). WMH is thought to disrupt white matter tracts and result in reorganization of functional brain patterns (Reijmer et al., 2015; De Marco et al., 2017). In the present study, since the 2 


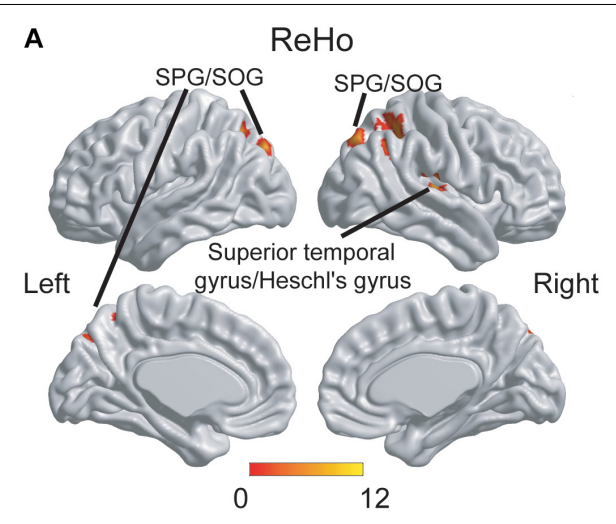

C
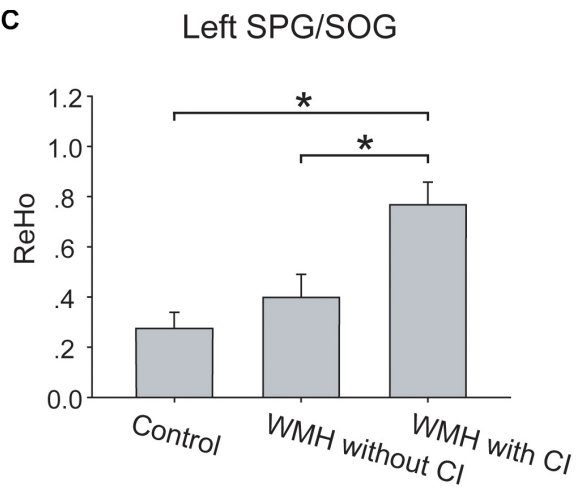

B Right superior temporal gyrus /Heschl's gyrus

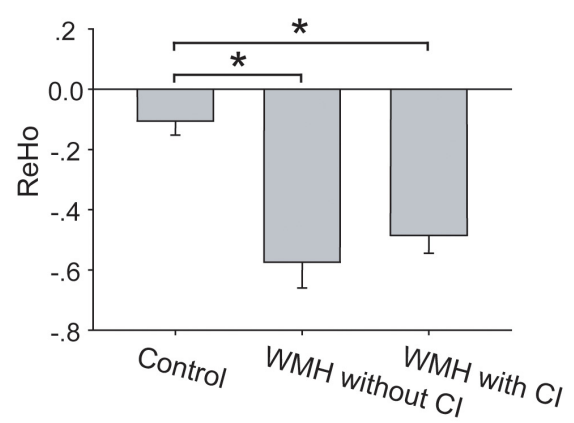

D Right SPG/SOG

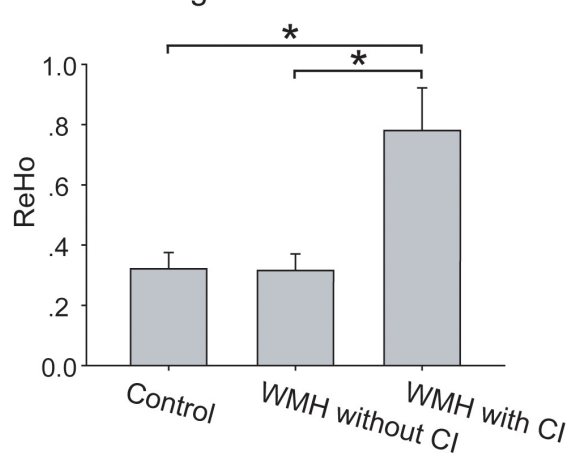

FIGURE 2 | The group differences of ReHo. (A) The group differences of ReHo were shown in the right superior temporal gyrus/Heschl's gyrus, left SPG/SOG and right SPG/SOG. (B) In the right superior temporal gyrus/Heschl's gyrus, both the WMH with $\mathrm{Cl}$ group and the WMH without $\mathrm{Cl}$ group displayed lower ReHo than the control group. (C,D) In the left SPG/SOG and right SPG/SOG, the WMH with Cl group showed higher ReHo than both the WMH without Cl group and the control group. ReHo values have been normalized by subtracting the mean voxel-wise ReHo obtained for the entire brain, and then dividing the resultant value by the standard deviation. The thresholds were set at a corrected $P<0.01$, determined by Monte Carlo simulation for multiple comparisons (voxel-wise $P<0.01$, FWHM $=6.9 \mathrm{~mm}$, cluster size $>1782 \mathrm{~mm}^{3}$ ). The color bars are presented with $F$-values. ${ }^{*} P<0.05, \mathrm{Cl}$, cognitive impairment; ReHo, Regional Homogeneity; SPG, superior parietal gyrus; SOG, superior occipital gyrus; WMH, white matter hyperintensities.

WMH groups had comparable WMH burden, the differences of functional patterns (i.e., ReHo and FC patterns) between the two groups could not be due to the difference of WMH burden, but could be related to the cognitive differences between the two groups. In the present study, higher ReHo in the SPG/SOG and higher FC of the SPG/SOG with parietal and frontal regions happened in the WMH with $\mathrm{CI}$ group, suggesting that the $\mathrm{CI}$ could be related to higher local coherence of activities in the SPG/SOG and higher connectivity between the SPG/SOG and parietal and frontal regions. Furthermore, the correlative analyses confirmed the results above.

In the present study, the WMH with $\mathrm{CI}$ group displayed poor performances in TMT-B, Stroop-B and Stroop-C tests, and increased ReHo in the SPG/SOG, suggesting that regional activities in parietal and occipital cortex were related to executive function in WMH with CI subjects. Parietal and occipital cortex plays a major role in the maintenance of normal cognition, including decision making, working memory, spatial updating and sensory attention (Medendorp et al., 2007; Tuladhar et al., 2007; Sulpizio et al., 2016), most of which are related to executive function. Furthermore, the maintenance of brain function relies on multiple brain areas that connect and interact with each other to serve different functions (Power et al., 2011). A balance between regional specialization and global integration is of vital importance for brain function (Tononi et al., 1998). A recent study found decreased FC of the default mode network and central executive network in subjects with both WHM and dementia (Kim et al., 2016). Another study investigated posterior cingulate cortex connectivity in $\mathrm{WMH}$ subjects with vascular cognitive impairment, no dementia, and showed both decreased FC with extensive regions and increased FC with parietal and temporal regions (Sun et al., 2011). In the present study, higher FC between the SPG/SOG and parietal and frontal regions were shown in the $\mathrm{WMH}$ with $\mathrm{CI}$ group. Higher FC of the left SPG/SOG with frontal regions were significantly associated with less worse executive dysfunction in WMH with CI subjects, suggesting a compensatory effect.

The mechanisms underlying the link between the functional alterations and $\mathrm{CI}$ in WMH subjects could be explained with a prominent cognitive model named "the scaffolding theory of aging and cognition (STAC)" (Reuter-Lorenz and Park, 2014). According to the STAC, cognitive decline is a consequence of 


\section{A}

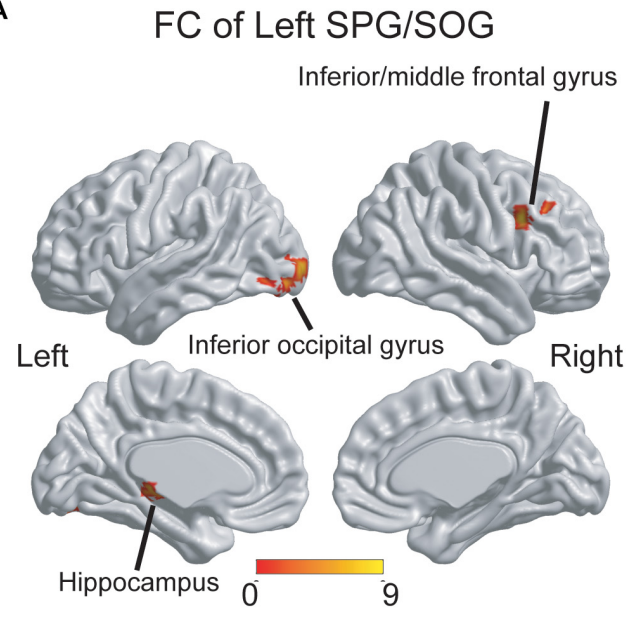

B

\section{FC of Right SPG/SOG}

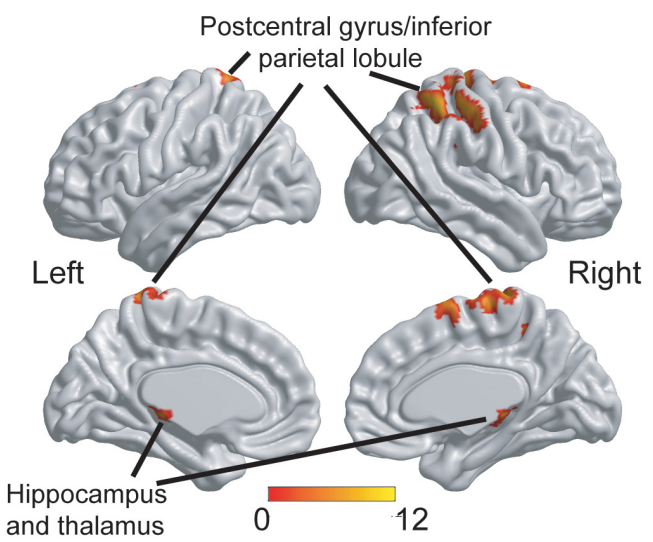

FIGURE 3 | The group differences of FC patterns of the bilateral SPG/SOG. (A) Significant group differences of FC of the left SPG/SOG were shown in frontal, temporal and occipital regions. (B) Significant group differences of FC of the right SPG/SOG were shown in parietal, temporal, and thalamus regions. The thresholds were set at a corrected $P<0.01$, determined by Monte Carlo simulation for multiple comparisons (voxel-wise $P<0.01$, FWHM $=7.4 \mathrm{~mm}$, cluster size $>2160 \mathrm{~mm}^{3}$ ). The color bars are presented with $F$-values. FC, functional connectivity; SPG, superior parietal gyrus; SOG, superior occipital gyrus.

"neural degradation," “compensatory scaffolding," and life-course factors. The neural degradation is thought to cause cognitive decline and is categorized as "neural challenges" and "functional deterioration." The former refers to structural changes in the brain, including white matter damages (Reuter-Lorenz and Park, 2014), and the latter is indicators of maladaptive brain activity such as dedifferentiation of activities in visual areas (Park et al., 2004; Voss et al., 2008). In the present study, although the WMH with $\mathrm{CI}$ group and the $\mathrm{WMH}$ without CI group had comparable WMH burden, only the WMH with CI group displayed higher ReHo, i.e., higher local coherence of activities, in the SPG/SOG. The higher local coherence of activities could be one of indicators of maladaptive brain activity, and suggest functional deterioration in the WMH with CI group. This could be one of inducing factors for CI in $\mathrm{WMH}$ subjects. On the other hand, these negative indices might induce the onset of compensatory scaffolding that refers to compensatory reallocation or recruitment of cognitive resources or supplementary neural circuitry (Greenwood, 2007; Cramer et al., 2011; Reuter-Lorenz and Park, 2014). Compensatory scaffolding counteracts or ameliorates the damage effect of neural degradation. The higher FC of the SPG/SOG with parietal and

TABLE 2 | Brain regions with group differences of ReHo or FC.

\begin{tabular}{|c|c|c|c|c|c|}
\hline Items & $\begin{array}{l}\text { Brain regions with group } \\
\text { differences }\end{array}$ & BA & Peak MNI coordinates $x, y, z(m m)$ & Peak $F$-value & Cluster size ( $m$ \\
\hline \multicolumn{6}{|l|}{ ReHo } \\
\hline & $\begin{array}{l}\text { Right superior temporal } \\
\text { gyrus/Heschl's gyrus }\end{array}$ & 48 & $42,-51,39$ & 10.98 & 2079 \\
\hline & Left SPG/SOG & 5,7 & $-18,-60,51$ & 9.52 & 2268 \\
\hline & Right SPG/SOG & 7,19 & $24,-68,36$ & 11.15 & 1944 \\
\hline \multicolumn{6}{|c|}{ FC of Left SPG/SOG } \\
\hline & $\begin{array}{l}\text { Right inferior/middle frontal } \\
\text { gyrus }\end{array}$ & 44,48 & $43,14,30$ & 8.59 & 2430 \\
\hline & Left inferior occipital gyrus & 19 & $-38,-79,-14$ & 7.95 & 2862 \\
\hline & Left hippocampus & 37 & $-24,-27,-6$ & 8.10 & 2241 \\
\hline \multicolumn{6}{|c|}{ FC of Right SPG/SOG } \\
\hline & $\begin{array}{l}\text { Bilateral postcentral } \\
\text { gyrus/inferior parietal lobule }\end{array}$ & $1-7,40$ & $9,-33,75$ & 11.67 & 14634 \\
\hline & $\begin{array}{l}\text { Bilateral hippocampus and } \\
\text { thalamus }\end{array}$ & 27 & $-14,-35,9$ & 8.23 & 2295 \\
\hline
\end{tabular}

The thresholds were set at a corrected $P<0.01$, determined by Monte Carlo simulation for multiple comparisons (voxel-wise $P<0.01$, for ReHo analysis, $F W H M=6.9 \mathrm{~mm}$, cluster size $>1782 \mathrm{~mm}^{3}$, and for FC analysis, FWHM $=7.4 \mathrm{~mm}$, cluster size $>2160 \mathrm{~mm}^{3}$ ). BA, Brodmann's area; MNI, Montreal Neurological Institute; FC, functional connectivity; SPG, superior parietal gyrus; SOG, superior occipital gyrus. 
TABLE 3 | FC data

\begin{tabular}{|c|c|c|c|c|c|c|}
\hline \multirow[t]{2}{*}{ Seed } & \multirow[t]{2}{*}{ Brain regions } & \multicolumn{3}{|c|}{ FC strength } & \multirow[t]{2}{*}{$\boldsymbol{F}$} & \multirow[t]{2}{*}{$P$-value } \\
\hline & & Control & WMH without Cl & WMH with Cl & & \\
\hline \multicolumn{7}{|c|}{ Left SPG/SOG } \\
\hline & Right inferior/middle frontal gyrus & $0.21 \pm 0.16$ & $0.15 \pm 0.15$ & $0.46 \pm 0.11^{a, b}$ & 12.07 & $<0.001$ \\
\hline & Left inferior occipital gyrus & $0.31 \pm 0.24$ & $0.39 \pm 0.19$ & $0.52 \pm 0.25^{a}$ & 6.74 & 0.005 \\
\hline & Left hippocampus & $0.12 \pm 0.13$ & $0.27 \pm 0.15^{\mathrm{a}}$ & $0.28 \pm 0.19^{a}$ & 7.05 & 0.002 \\
\hline \multicolumn{7}{|c|}{ Right SPG/SOG } \\
\hline & Bilateral postcentral gyrus/inferior parietal lobule & $0.41 \pm 0.11$ & $0.40 \pm 0.14$ & $0.69 \pm 0.18^{a, b}$ & 12.31 & $<0.001$ \\
\hline & Bilateral hippocampus and thalamus & $0.06 \pm 0.15$ & $0.21 \pm 0.15^{\mathrm{a}}$ & $0.21 \pm 0.18^{a}$ & 7.18 & $<0.001$ \\
\hline
\end{tabular}

The thresholds were set at a corrected $P<0.01$, determined by Monte Carlo simulation for multiple comparisons (voxel-wise $P<0.01$, for ReHo analysis, $F W H M=6.9 \mathrm{~mm}$, cluster size $>1782 \mathrm{~mm}^{3}$, and for $F C$ analysis, FWHM $=7.4 \mathrm{~mm}$, cluster size $>2160 \mathrm{~mm}^{3}$ ). ${ }^{a} P<0.05$, differs $^{2}$ from the control group; ${ }^{b} P<0.05$,

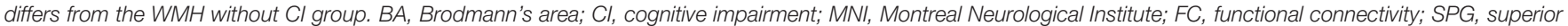
parietal gyrus; SOG, superior occipital gyrus.

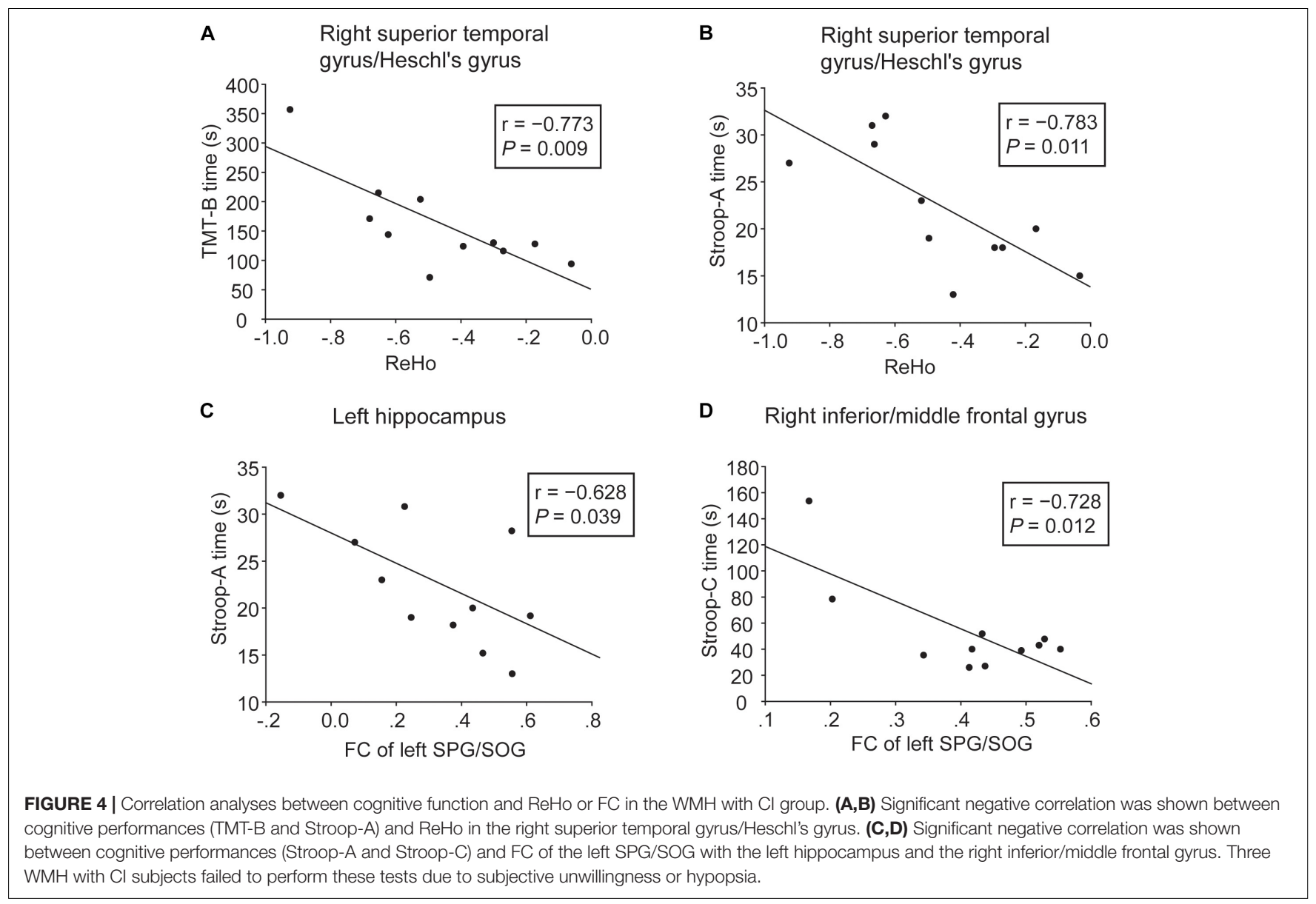

frontal regions in the $\mathrm{WMH}$ with $\mathrm{CI}$ group indicated enhanced functional communications between the SPG/SOG and other regions. The results of correlative analyses showed that the enhanced communications partly compensated for executive dysfunction in WMH with CI subjects. Thus, the higher ReHo in the SPG/SOG and the higher FC of the SPG/SOG with parietal and frontal regions could represent functional deterioration and compensatory scaffolding, respectively, during the development of CI in WMH subjects.
Most of previous fMRI studies on WMH subjects investigated the effects of WMH burden on brain activities or connectivity. A recent study found that $\mathrm{WMH}$ burden modulated brain connectivity in healthy subjects, i.e., high WMH burden was associated with increased FC of default mode network and salience network with temporal cortex and parietal cortex, respectively (De Marco et al., 2017). A study investigated the spatial associations of intrinsic connectivity contrast with WMH volume in elderly subjects, and found that significant associations 
were detected between intrinsic connectivity contrast of SOG and WMH volume in subcortical white matter (Shi et al., 2017). A task fMRI study on healthy aging showed that greater WMH volume was associated with increased frontal activation and decreased frontal FC during performing a spatial search task (Lockhart et al., 2015). All these findings supported altered functional brain patterns across frontal, parietal, temporal and occipital regions in $\mathrm{WMH}$ subjects and also suggested a compensatory functional enhancement underlying the maintenance of normal cognition in WMH subjects. Consistent with these findings, the present study confirmed altered regional activities in parietal, occipital and temporal regions and increased FC with frontal, parietal and temporal regions in WMH subjects. Notably, the present study was performed on both WMH with and without CI subjects. The WMH without CI group displayed decreased ReHo in temporal regions and increased FC of the SPG/SOG with hippocampus. The increased FC with hippocampus might reflect a compensatory functional enhancement. In addition, compensatory functional enhancements were also shown in WMH with CI subjects. Thus, the present findings extended the compensatory functional enhancements on $\mathrm{WMH}$ subjects with mild CI.

Some limitations should be addressed. First, the sample size in the present study is small, especially for WMH subjects, and $3 \mathrm{WMH}$ with $\mathrm{CI}$ subjects even failed to perform some of neuropsychological tests. The findings should be validated in a larger sample. We are continuing to recruit new participants with WMH to validate our findings. Second, due to the small sample size and a large number of correlation analyses between functional brain alterations and cognition, the results of correlative analyses would lose significance after correcting for the Bonferroni correction principle. Thus, the present findings should be treated with caution.

\section{CONCLUSION}

In conclusion, with comparable WMH burden, WMH subjects with CI have higher local coherence of activities in the SPG/SOG

\section{REFERENCES}

Aisen, P. S., Petersen, R. C., Donohue, M. C., Gamst, A., Raman, R., Thomas, R. G., et al. (2010). Clinical core of the Alzheimer's disease neuroimaging initiative: progress and plans. Alzheimers Dem. 6, 239-246.

Brickman, A. M., Zahodne, L. B., Guzman, V. A., Narkhede, A., Meier, I. B., Griffith, E. Y., et al. (2015). Reconsidering harbingers of dementia: progression of parietal lobe white matter hyperintensities predicts Alzheimer's disease incidence. Neurobiol. Aging 36, 27-32. doi: 10.1016/j.neurobiolaging.2014. 07.019

Chen, H., Uddin, L. Q., Guo, X., Wang, J., Wang, R., Wang, X., et al. (2019). Parsing brain structural heterogeneity in males with autism spectrum disorder reveals distinct clinical subtypes. Hum. Brain Mapp. 40, 628-637. doi: 10.1002/hbm. 24400

Cramer, S. C., Sur, M., Dobkin, B. H., O’Brien, C., Sanger, T. D., Trojanowski, J. Q., et al. (2011). Harnessing neuroplasticity for clinical applications. Brain $134(\mathrm{Pt}$ 6), 1591-1609. doi: 10.1093/brain/awr039

de Leeuw, F. E., de Groot, J. C., Achten, E., Oudkerk, M., Ramos, L. M., Heijboer, R., et al. (2001). Prevalence of cerebral white matter lesions in elderly people: a and higher connectivity of the SPG/SOG with parietal and frontal regions than $\mathrm{WMH}$ subjects without $\mathrm{CI}$. The findings provide novel insights into the functional alterations underlying the cognitive variety in WMH subjects and shed light on the investigation of surrogate markers for $\mathrm{CI}$ in WMH subjects.

\section{ETHICS STATEMENT}

This study was carried out in accordance with the recommendations of the Drum Tower Hospital Research Ethics Committee. All subjects gave written informed consent in accordance with the Declaration of Helsinki. The protocol was approved by the Drum Tower Hospital Research Ethics Committee.

\section{AUTHOR CONTRIBUTIONS}

YX designed the study and revised the manuscript. QY and XC carried out the data collection and data analysis, and wrote the manuscript. RQ, LH, DY, and RL carried out the data collection. FB and BZ discussed the study. All authors approved the final version of the manuscript.

\section{FUNDING}

This work was supported by the National Key Research and Development Program of China (Grant Nos. 2016YFC1300504 and 2016YFC0901004), the National Natural Science Foundation of China (Grant Nos. 81801060 and 81630028), the Fundamental Research Funds for the Central Universities (Grant No. YG1805070+021414380430), the Key Research and Development Program of Jiangsu Province of China (Grant No. BE2016610), the Jiangsu Province Key Medical Discipline (Grant No. ZDXKA2016020), and the Jiangsu Provincial Key Medical Talents (Grant No. ZDRCA2016085).

population based magnetic resonance imaging study. the rotterdam scan study. J. Neurol. Neurosurg. Psychiatry 70, 9-14. doi: 10.1136/jnnp.70.1.9

De Marco, M., Manca, R., Mitolo, M., and Venneri, A. (2017). White matter hyperintensity load modulates brain morphometry and brain connectivity in healthy adults: a neuroplastic mechanism? Neural Plast 2017:4050536. doi: $10.1155 / 2017 / 4050536$

Debette, S., and Markus, H. S. (2010). The clinical importance of white matter hyperintensities on brain magnetic resonance imaging: systematic review and meta-analysis. BMJ 341:c3666. doi: 10.1136/bmj.c3666

Diciotti, S., Orsolini, S., Salvadori, E., Giorgio, A., Toschi, N., Ciulli, S., et al. (2017). Resting state fMRI regional homogeneity correlates with cognition measures in subcortical vascular cognitive impairment. J. Neurol. Sci. 373, 1-6. doi: 10.1016/j.jns.2016.12.003

Gouw, A. A., Seewann, A., van der Flier, W. M., Barkhof, F., Rozemuller, A. M., Scheltens, P., et al. (2011). Heterogeneity of small vessel disease: a systematic review of MRI and histopathology correlations. J. Neurol. Neurosurg. Psychiatry 82, 126-135. doi: 10.1136/jnnp.2009.204685

Greenwood, P. M. (2007). Functional plasticity in cognitive aging: review and hypothesis. Neuropsychology 21, 657-673. doi: 10.1037/0894-4105.21.6.657 
Guo, X., Duan, X., Suckling, J., Chen, H., Liao, W., and Cui, Q. (2019). Partially impaired functional connectivity states between right anterior insula and default mode network in autism spectrum disorder. Hum. Brain Mapp. 40, 1264-1275. doi: 10.1002/hbm.24447

Jack, C. R. Jr., Wiste, H. J., Vemuri, P., Weigand, S. D., Senjem, M. L., Zeng, G., et al. (2010). Brain beta-amyloid measures and magnetic resonance imaging atrophy both predict time-to-progression from mild cognitive impairment to Alzheimer's disease. Brain 133, 3336-3348. doi: 10.1093/brain/awq277

Kim, H. J., Cha, J., Lee, J. M., Shin, J. S., Jung, N. Y., Kim, Y. J., et al. (2016). Distinctive resting state network disruptions among alzheimer's disease, subcortical vascular dementia, and mixed dementia patients. J. Alzheimers Dis. 50, 709-718. doi: 10.3233/JAD-150637

Lampe, L., Kharabian-Masouleh, S., Kynast, J., Arelin, K., Steele, C. J., Loffler, M., et al. (2019). Lesion location matters: the relationships between white matter hyperintensities on cognition in the healthy elderly. J. Cereb. Blood Flow Metab. 39, 36-43. doi: 10.1177/0271678X17740501

Lockhart, S. N., Luck, S. J., Geng, J., Beckett, L., Disbrow, E. A., Carmichael, O., et al. (2015). White matter hyperintensities among older adults are associated with futile increase in frontal activation and functional connectivity during spatial search. PLoS One 10:e0122445. doi: 10.1371/journal.pone.0122445

Longstreth, W. T. Jr., Manolio, T. A., Arnold, A., Burke, G. L., Bryan, N., Jungreis, C. A., et al. (1996). Clinical correlates of white matter findings on cranial magnetic resonance imaging of 3301 elderly people. the cardiovascular health study. Stroke 27, 1274-1282. doi: 10.1161/01.str.27.8.1274

Medendorp, W. P., Kramer, G. F., Jensen, O., Oostenveld, R., Schoffelen, J. M., and Fries, P. (2007). Oscillatory activity in human parietal and occipital cortex shows hemispheric lateralization and memory effects in a delayed double-step saccade task. Cereb. Cortex 17, 2364-2374. doi: 10.1093/cercor/bhl145

Pantoni, L., Basile, A. M., Pracucci, G., Asplund, K., Bogousslavsky, J., Chabriat, H., et al. (2005). Impact of age-related cerebral white matter changes on the transition to disability - the LADIS study: rationale, design and methodology. Neuroepidemiology 24, 51-62. doi: 10.1159/00008 1050

Park, D. C., Polk, T. A., Park, R., Minear, M., Savage, A., and Smith, M. R. (2004). Aging reduces neural specialization in ventral visual cortex. Proc. Natl. Acad. Sci. U.S.A. 101, 13091-13095. doi: 10.1073/pnas.0405148101

Power, J. D., Barnes, K. A., Snyder, A. Z., Schlaggar, B. L., and Petersen, S. E. (2012). Spurious but systematic correlations in functional connectivity MRI networks arise from subject motion. Neuroimage 59, 2142-2154. doi: 10.1016/j. neuroimage.2011.10.018

Power, J. D., Cohen, A. L., Nelson, S. M., Wig, G. S., Barnes, K. A., Church, J. A., et al. (2011). Functional network organization of the human brain. Neuron 72 , 665-678.

Prins, N. D., van Dijk, E. J., den Heijer, T., Vermeer, S. E., Jolles, J., Koudstaal, P. J., et al. (2005). Cerebral small-vessel disease and decline in information processing speed, executive function and memory. Brain 128(Pt 9), 2034-2041. doi: 10.1093/brain/awh553

Reijmer, Y. D., Schultz, A. P., Leemans, A., O'Sullivan, M. J., Gurol, M. E., Sperling, R., et al. (2015). Decoupling of structural and functional brain connectivity in older adults with white matter hyperintensities. Neuroimage 117, 222-229. doi: 10.1016/j.neuroimage.2015.05.054

Reuter-Lorenz, P. A., and Park, D. C. (2014). How does it STAC up? Revisiting the scaffolding theory of aging and cognition. Neuropsychol. Rev. 24, 355-370. doi: 10.1007/s11065-014-9270-9

Satterthwaite, T. D., Elliott, M. A., Gerraty, R. T., Ruparel, K., Loughead, J., Calkins, M. E., et al. (2013). An improved framework for confound regression and filtering for control of motion artifact in the preprocessing of restingstate functional connectivity data. Neuroimage 64, 240-256. doi: 10.1016/j. neuroimage.2012.08.052

Schmidt, P., Gaser, C., Arsic, M., Buck, D., Forschler, A., Berthele, A., et al. (2012). An automated tool for detection of FLAIR-hyperintense white-matter lesions in multiple sclerosis. Neuroimage 59, 3774-3783. doi: 10.1016/j.neuroimage.2011. 11.032
Schmidt, R., Berghold, A., Jokinen, H., Gouw, A. A., van der Flier, W. M., Barkhof, F., et al. (2012). White matter lesion progression in LADIS: frequency, clinical effects, and sample size calculations. Stroke 43, 2643-2647. doi: 10.1161/ strokeaha.112.662593

Shi, L., Miao, X., Lou, W., Liu, K., Abrigo, J., Wong, A., et al. (2017). The spatial associations of cerebral blood flow and spontaneous brain activities with white matter hyperintensities-an exploratory study using multimodal magnetic resonance imaging. Front. Neurol. 8:593. doi: 10.3389/fneur.2017.00593

Sudo, F. K., Alves, C. E., Alves, G. S., Ericeira-Valente, L., Tiel, C., Moreira, D. M., et al. (2013). White matter hyperintensities, executive function and global cognitive performance in vascular mild cognitive impairment. Arq. Neuropsiquiatr. 71, 431-436. doi: 10.1590/0004-282X2013 0057

Sulpizio, V., Committeri, G., Lambrey, S., Berthoz, A., and Galati, G. (2016). Role of the human retrosplenial cortex/parieto-occipital sulcus in perspective priming. Neuroimage 125, 108-119. doi: 10.1016/j.neuroimage.2015.10.040

Sun, Y. W., Qin, L. D., Zhou, Y., Xu, Q., Qian, L. J., Tao, J., et al. (2011). Abnormal functional connectivity in patients with vascular cognitive impairment, no dementia: a resting-state functional magnetic resonance imaging study. Behav. Brain Res. 223, 388-394. doi: 10.1016/j.bbr.2011.05.006

Tononi, G., Edelman, G. M., and Sporns, O. (1998). Complexity and coherency: integrating information in the brain. Trends Cogn. Sci. 2, 474-484. doi: 10.1016/ s1364-6613(98)01259-5

Tuladhar, A. M., ter Huurne, N., Schoffelen, J. M., Maris, E., Oostenveld, R., and Jensen, O. (2007). Parieto-occipital sources account for the increase in alpha activity with working memory load. Hum. Brain Mapp. 28, 785-792. doi: 10.1002/hbm.20306

van Dijk, E. J., Prins, N. D., Vrooman, H. A., Hofman, A., Koudstaal, P. J., and Breteler, M. M. (2008). Progression of cerebral small vessel disease in relation to risk factors and cognitive consequences: rotterdam scan study. Stroke 39, 2712-2719. doi: 10.1161/STROKEAHA.107.513176

Voss, M. W., Erickson, K. I., Chaddock, L., Prakash, R. S., Colcombe, S. J., Morris, K. S., et al. (2008). Dedifferentiation in the visual cortex: an fMRI investigation of individual differences in older adults. Brain Res. 1244, 121-131. doi: 10.1016/ j.brainres.2008.09.051

Xu, X., Gao, Y., Liu, R., Qian, L., Chen, Y., Wang, X., et al. (2018). Progression of white matter hyperintensities contributes to lacunar infarction. Aging Dis. 9, 444-452. doi: 10.14336/AD.2017.0808

Ye, Q., Su, F., Gong, L., Shu, H., Liao, W., Xie, C., et al. (2017). Divergent roles of vascular burden and neurodegeneration in the cognitive decline of geriatric depression patients and mild cognitive impairment patients. Front. Aging Neurosci. 9:288. doi: 10.3389/fnagi.2017.00288

Zang, Y., Jiang, T., Lu, Y., He, Y., and Tian, L. (2004). Regional homogeneity approach to fMRI data analysis. Neuroimage 22, 394-400. doi: 10.1016/j. neuroimage.2003.12.030

Zhuang, F. J., Chen, Y., He, W. B., and Cai, Z. Y. (2018). Prevalence of white matter hyperintensities increases with age. Neural Regen. Res. 13, 2141-2146.

Zuo, X. N., Xu, T., Jiang, L., Yang, Z., Cao, X. Y., He, Y., et al. (2013). Toward reliable characterization of functional homogeneity in the human brain: preprocessing, scan duration, imaging resolution and computational space. Neuroimage 65, 374-386. doi: 10.1016/j.neuroimage.2012.10.017

Conflict of Interest Statement: The authors declare that the research was conducted in the absence of any commercial or financial relationships that could be construed as a potential conflict of interest.

Copyright $\odot 2019$ Ye, Chen, Qin, Huang, Yang, Liu, Zhang, Bai and Xu. This is an open-access article distributed under the terms of the Creative Commons Attribution License (CC BY). The use, distribution or reproduction in other forums is permitted, provided the original author(s) and the copyright owner(s) are credited and that the original publication in this journal is cited, in accordance with accepted academic practice. No use, distribution or reproduction is permitted which does not comply with these terms. 